\title{
Characteristics and functions of murine cutaneous dendritic cells: a synopsis of recent developments
}

\author{
MC Udey ${ }^{1}$ and K Nagao ${ }^{1,2}$
}

\begin{abstract}
Cutaneous accessory cells include dendritic cells (DCs) and macrophages. Heterogeneity, plasticity, and responsiveness to local environmental cues are hallmarks of both types of cells. Until recently, results of studies of cells that had been extracted from tissues or propagated in vitro provided the foundation for most conceptual frameworks. The availability of a variety of spontaneously occurring and genetically engineered mice has facilitated in vivo studies that have provided new insights into the developmental and functional aspects of DCs in skin and other tissues. In several instances, results of these in vivo studies have been very surprising. Existing paradigms have been modified or debunked, and new hypotheses have been generated. We can anticipate that detailed understanding of the biology of individual cutaneous accessory cells and their relationships with each other will continue to accumulate as these types of studies are actively pursued.
\end{abstract}

\section{INTRODUCTION}

Skin is perhaps the most extensively studied interface organ. Immunologists have shown interest in skin for decades and many prominent investigative dermatologists have been, and are, immunologists. Observational studies in patients and normal individuals have been complemented by experimental studies, initially in guinea-pigs and more recently in mice. Reductionist approaches that, by necessity, focused on cells that were extracted from tissues or that were propagated in the laboratory have provided much of the information on which our understanding of cutaneous immunophysiology is based.

Dendritic cells (DCs) and macrophages comprise the bulk of accessory cells in both normal and inflamed skin. The former function as sentinels linking innate and adaptive immunity whereas the latter act as scavengers of apoptotic cells and microbes, and participate actively in reparative processes as well. Both types of cells are infrequently represented, especially in normal skin, and isolation is not trivial or necessarily atraumatic. Increasingly, it is also recognized that both DCs and macrophages are heterogenous, even in a single tissue such as the skin, and that their properties are influenced by their local environments. Thus, ex vivo studies of skin-derived DCs and macrophages are laborious and results of experiments with these cells need to be carefully interpreted. Studies of cells that have been propagated from skin-associated or bone marrow-derived precursors represent an alternative to studies of cells that are extracted from the skin, but this approach also has limitations. The fact that one cannot easily reconstitute a bona fide "skin environment" in tissue culture is compounded by incomplete knowledge of characteristics of the cells that one is trying to recapitulate.

Recently, it has become possible to test basic tenets of cutaneous accessory cell biology by studying carefully selected (or generated) mouse mutants and experimental model systems. Results of some of these experiments have been paradigm shifting. This brief review will highlight some recent work in mice that has reinforced results of previous studies, reshaped existing concepts, and, in some cases, led to entirely new hypotheses. The focus will be on DCs rather than macrophages because much of the new information relates to this leukocyte population. The reader is referred to several recent reviews for more detailed information. $^{1-3}$

\section{DEFINED SUBSETS OF CUTANEOUS DENDRITIC CELLS}

Paul Langerhans, an Austrian medical student, identified rare cells with an unexplained affinity for gold salts and a dendritic morphology in the epidermis of human skin more than 100 years ago. Descriptive studies emphasizing morphological and histochemical characteristics suggested that Langerhans cells (LCs) were related to neurons or melanocytes. That LCs were leukocytes was definitively demonstrated in bone marrow transplantation studies, ${ }^{4,5}$ and their ability to function as accessory

\footnotetext{
${ }^{1}$ Dermatology Branch, Center for Cancer Research, National Cancer Institute, NIH, Bethesda, Maryland, USA. ${ }^{2}$ Present address: Department of Dermatology, Keio University, Tokyo, Japan. Correspondence: MC Udey (udey@helix.nih.gov)

Received 25 July 2008; accepted 31 July 2008; published online 10 September 2008. doi:10.1038/mi.2008.37
} 
cells was subsequently characterized extensively in in vitro studies and in studies in which isolated LCs were adoptively transferred after a variety of manipulations. Working with Steinman ${ }^{6}$, Schuler showed that LC represented the epidermal contingent of the DC lineage, a subset of cells that Steinman and co-workers ${ }^{7}$ had identified and characterized in lymphoid tissues, including lymph nodes and spleen. This observation heralded the beginning of a new era of LC biology.

Langerhans cells came to be regarded as the prototypic "immature" tissue DCs and, for many years, investigators focused on these cells to the relative exclusion of other accessory cells in the skin. LC in epidermis (actually LC studied ex vivo immediately after isolation) exhibited phagocytic and vigorous pinocytic activities, efficiently processed protein antigens, expressed low levels of major histocompatibility complex antigens and costimulatory molecules, and were poor stimulators of naive $\mathrm{T}$ cells. LC that had been induced to migrate to draining lymph nodes (actually LC that had been cultured in vitro in granulocyte monocyte colony-stimulating factor-containing media or LC that migrated out of skin explants ex vivo) exhibited decreased antigen acquisition and processing activity and increased antigen presentation function coincident with increased surface expression of major histocompatibility complex antigens (especially, major histocompatibility complex class II) and costimulatory molecules (especially CD40 and CD86).

On the basis of these types of studies, it was hypothesized that LCs captured exogenous antigens, were activated by microbeassociated constituents or proinflammatory cytokines, and migrated to lymph nodes where they initiated effector T-cell responses. Results of a variety of adoptive transfer and other in vivo experiments were consistent with this paradigm. It was hypothesized that similar trafficking of LC occurred in the absence of an inflammatory stimulus and that in this instance peripheral tolerance could result because of induction of T-cell anergy or selective stimulation of regulatory T cells. ${ }^{8}$

As information about LCs and other DCs has accumulated, it has become clear that LCs are distinct in several ways. They are uniquely dependent on transforming growth factor (TGF) $\beta 1$ for development, localization, and survival and they persist in unperturbed or minimally perturbed epidermis for long periods of time (weeks to months). They also express Langerin, a C-type lectin that localizes to an endocytic vesicle with a distinctive morphology and that induces the formation of these structures (termed Birbeck granules) after expression in fibroblasts. Although the significance of Langerin expression by LC is not fully appreciated, ${ }^{9}$ selective expression of Langerin by LC has been exploited to develop mice that have been of great utility. These include transgenic mice that express the diphtheria toxin A subunit (DTA) or the recombinase cre downstream of a regulatory region derived from the human Langerin promoter, ${ }^{10}$ as well as knock-in mice in which cDNAs encoding the diphtheria toxin receptor (DTR) have been inserted into the endogenous Langerin locus. ${ }^{11,12}$ LCs are not found in the epidermis of Langerin-DTA mice and they can be conditionally deleted in Langerin-DTR mice after administration of a singe dose of DT. By analogy, cre is selectively expressed in the LC of Langerin- cre mice allowing for lineage-selective deletion of genes that are flanked with lox p sites. Recently, it has been recognized that, in mice, Langerin expression is not restricted to LC. In addition to LC, Langerin is readily detected in CD8 $\alpha$-expressing lymph node DC (in some strains) and a population of dermal DC as well. ${ }^{13-15}$

EpCAM (epithelial cell adhesion molecule) represents another protein that, among murine DCs, is selectively expressed by LC. ${ }^{16}$ Recent studies indicate that EpCAM is not expressed by Langerin + dermal DC, ${ }^{14}$ allowing skin-derived epidermal LC and Langerin-expressing dermal DC to be readily distinguished. As EpCAM is expressed by LCs that have migrated to lymph nodes, the argument that Langerin + dermal DCs represent LCs that are in transit from epidermis to lymph node does not seem to be tenable (see below). As some dermal DCs express neither Langerin nor EpCAM, there appear to be at least three definable CD11c-expressing DC subsets in normal murine skin: Langerin + EpCAM + epidermal LC, Langerin + EpCAM - dermal DC, and Langerin - EpCAM - dermal DC. In addition to these cells, migratory Langerin + EpCAM + LC can be identified in the dermis. It seems likely that additional DC sub-populations will be identified as new surface markers of interest that are identified and studied. The existence of additional and perhaps novel DC sub-populations in inflamed mouse skin, analogous to FcR- $\varepsilon$-expressing inflammatory DC and tumor necrosis factor- and inducible nitric oxide synthaseproducing DCs that have been identified in human atopic and psoriatic skin for example, is also very likely.

\section{LINEAGE RELATIONSHIPS BETWEEN CUTANEOUS DENDRITIC CELLS}

As indicated above, LCs have several characteristics that distinguish them from other DCs. Recently, considerable effort has been focused on defining lineage relationships between LCs and other DCs in skin and in other tissues, and identifying immediate precursors of LC. A variety of in vivo approaches employing carefully selected mutant mice have been informative.

The initial bone marrow transplantation studies that demonstrated that LCs were leukocytes involved donor and recipient mice that were mismatched at a number of histocompatibility loci as a result of parent into $\mathrm{F} 1$ reconstitutions. ${ }^{4,5}$ In these experiments, population of recipient epidermis by donorderived LC occurred over a period of weeks. The issue of turnover of LC in the steady state was subsequently readdressed by creating congenic bone marrow chimeras and parabiotic mice. ${ }^{17}$ In these experiments, recipient LC persisted in epidermis for more than 1 year. Recently, analogous experiments have been performed using donors and recipients that differed at major and minor loci and $\mathrm{T}$ cell-depleted bone marrow as a source of hematopoetic stem cells. ${ }^{18}$ In this setting, recipient LC persisted in the epidermis of chimeric mice for months, and population of epidermis with donor-derived LC was correspondingly delayed. Interestingly, deliberate induction of graft vs. host disease (GvHD) in recipients (by administering small numbers of donor T cells) or treatment of chimeric mice with ultraviolet radiation led to rapid accumulation of donor-derived LC in the 
epidermis. In retrospect, it seems likely that subclinical GvHD occurred in the initial bone marrow transplantation studies and that this facilitated rapid population of recipient skin with LC from the donors. Thus, although the initial studies were flawed, they led to the conclusion that LCs were of bone marrow origin. It is interesting to think about what conclusion would have been drawn if LC had appeared to be radioresistant in the initial studies. Most likely, it would have been incorrectly concluded that LCs were not leukocytes.

The demonstration that LCs were absent from the skin of TGF $\beta 1$ knockout mice also provided insight into an important aspect of LC development. ${ }^{19}$ Follow-up bone marrow transplantation studies involving TGF $\beta 1$-deficient donors and wild-type recipients indicated that TGF $\beta 1$ from recipient cells (presumably keratinocytes) could support LC development. ${ }^{20}$ As the TGF $\beta 1$-deficient mice that were available at the time were on a mixed C57BL/6 and 129Sv background, it is likely that subclinical GvHD also occurred in these experiments. The involvement of TGF $\beta 1$ in LC development has recently been revisited using newly generated mice in experiments that allow more straightforward interpretations. ${ }^{21}$ In these experiments, the Langerin promoter was used to drive the expression of the recombinase cre selectively in LC. Crossing Langerin-cre mice with mice with a floxed TGF $\beta$ RII allele gave rise to mice that were LC deficient. This result is consistent with the concept that LC must be TGF $\beta 1$ responsive to develop, localize in, and/or survive in the epidermis. This result was expected in the sense that it is entirely consistent with earlier results, but these studies do rigorously exclude a secondary effect of TGF $\beta 1$ deficiency (e.g., the systemic inflammatory reaction that develops in TGF $\beta 1$-deficient mice) as a cause of LC deficiency. An analogous approach was used to re-examine the question of the relevance of LC-extrinsic TGF $\beta 1$ in LC development. ${ }^{21}$ Langerin-cre mice were crossed with mice with a floxed TGF $\beta 1$ allele. Interestingly, these mice were also LC deficient-a result that is discordant with the previous bone marrow transplant experiment ${ }^{20}$ and is perhaps related to subclinical GvHD that may have been present in chimeric animals.

The studies described above, in conjunction with others, lead credence to the concept that the lineage derivations and/or the nature of precursors of LC, and perhaps other DC, is context dependent. Under steady-state conditions, LCs appear to derive from radioresistant cells that slowly proliferate within the skin. ${ }^{22}$ The precise nature of these precursors has yet to be defined, but the observation that M-CSFR-deficient mice are also LC deficient is consistent with the hypothesis that steady-state LC could be derived from monocytes. ${ }^{23}$ The characteristics of LC precursors in the setting of GvHD and/or ultraviolet-induced LC depletion and inflammation are better defined. In these situations, it appears that $\mathrm{Gr} 1+$ monocytes serve as precursors and that the actions of CCR2 and CCR6 ligands are required for recruitment. ${ }^{17,18}$ This conclusion is consistent with the results that indicate that cells with LC characteristics can develop from human CD14 + dermal cells. ${ }^{24}$ One possible caveat to the interpretation of these studies is that some were performed before the existence of Langerin + cells in the skin that are not LC was known. Thus, it is formally possible that some of these conclusions relate to recently described Langerin + dermal DC rather than LC. ${ }^{13-15}$

Discovery of expression of Langerin (previously the Lag antigen) by human LC preceded the description of the corresponding protein in murine LC, and the generation of useful antibodies for studies in mice lagged considerably. Development of antibodies reactive with mouse Langerin has allowed the heterogeneity of skin DC to be additionally explored, ${ }^{24}$ and three distinct subsets have been defined (see above). All DCs that are present in uninflamed epidermis are LCs, and no heterogeneity has been identified in this population. In the dermis, the existence of two DC populations has been confirmed (CD11c+ Langerin + EpCAM - dermal DC and CD11c + Langerin - dermal DC) and a third population (CD11c + Langerin + EpCAM + DC that represent LCs that are in transit from epidermis to lymph node) is likely to be present. On the basis of repopulation kinetics in chimeric mice and studies in parabiotic mice, it appears that LCs and Langerin + dermal DCs are not lineage related, but it is difficult to exclude this possibility with certainty. The possible lineage relationships between CD11c+ Langerin + dermal DC and CD11c+ Langerin - dermal DC are yet to be explored.

\section{NEW INSIGHTS INTO FUNCTIONAL ASPECTS OF CUTANEOUS ACCESSORY CELLS}

The recognition that skin DCs are diverse based on surface phenotypic and lineage characteristics has prompted a re-evaluation of their functional activities. The availability of mice that are, or that can be induced to become, deficient in selected DC sub-populations has allowed for functional studies that were heretofore impossible. Studies to date have focused on Langerinexpressing DCs because it is these cells that have been selectively targeted.

The results of the studies that utilize Langerin-DTA mice ${ }^{10}$ are easiest to interpret. These mice are devoid of epidermal LC, but for reasons that are unclear have normal contingents of Langerin + dermal DC and CD $8 \alpha+$ Langerin + lymph node DC. Langerin-DTA mice are indistinguishable from normal animals in the absence of provocation. In contact sensitivity experiments, Langerin-DTA mice exhibited enhanced responsiveness. ${ }^{10}$ These results indicate that LCs are not required for sensitization or elicitation phases of these CD8 T cell-dependent immune responses to epicutaneously applied antigens and that they may have a suppressive function instead. Interestingly, Langerin-DTA mice on an FVB/N genetic background have also been shown to be resistant to dimethyl benzanthracene/tetradecanoylphorbol-13-acetate induced skin tumorigenesis, suggesting that LCs may also have an immunosuppressive function in this setting. ${ }^{26}$

Langerin-DTA mice have also been used to assess the role of LC in skin graft rejection responses. ${ }^{27} \mathrm{C} 57 \mathrm{BL} / 6$ mice reject fullthickness FVB/N skin grafts independent of whether FVB/N skin grafts have LC. Female C57BL/6 mice also reject male C57BL/6 skin grafts from both Langerin-DTA and control mice. In analogy to the contact sensitivity experiments, the results 
of these skin-grafting experiments suggest that LCs are not required for the development of antigraft responses or expression of antigraft reactivity. Results were rather different when male $\mathrm{FVB} / \mathrm{N}$ skin was grafted onto female $\mathrm{FVB} / \mathrm{N}$ recipients. In this strain, the expected result was that female mice would not reject male skin grafts. This result was replicated when LCs were present in the grafts, but male $\mathrm{FVB} / \mathrm{N}$ skin grafts were rejected when they were obtained from LC-deficient mice. The mechanism that is responsible for this surprising result has not yet been elucidated.

The involvement of Langerin-expressing DC in skin-centered immune responses has also been studied in Langerin-DTR mice. These mice have normal numbers of LC and are indistinguishable from wild-type littermates until they are treated with DT. ${ }^{11,12}$ A single systemic treatment with DT results in virtually complete loss of LC within 24-48 h. In these mice, DT treatment also leads to the depletion of Langerin + dermal DC and Langerin + CD8 $\alpha+$ lymph node DC. ${ }^{13-15}$ LC depletion is sustained in DTtreated Langerin-DTR mice, and significant numbers of LC do not appear in the epidermis for weeks. In contrast, Langerin + dermal and lymph node DCs repopulate their relative compartments within a few days after DT administration. Thus, the differential repopulation kinetics of LC and Langerin + dermal DC must be taken into account when using these mice to try to elucidate the functions of either population in in vivo studies. Careful studies of appropriate models may provide insights into the functions of both sub-populations.

The earliest functional studies carried out with LangerinDTR mice were performed before Langerin + dermal DCs were described. ${ }^{11,12,28-30}$ As a result, the experimental design of some studies was not optimal and some conclusions may be incorrect. More recent studies that take Langerin + dermal DC into account have failed to document a function for LC in the priming of effector $\mathrm{T}$ cells that react with contact allergens or epicutaneously administered proteins. ${ }^{14,31}$ In contrast, these studies suggest that Langerin + dermal DC may be required for optimal responses. Interestingly, augmented immune responses analogous to those that have been seen in response to contact allergens in LangerinDTA mice have not been described in Langerin-DTR mice under any circumstances. One possible explanation for this is that LC may play an unappreciated role in immune homeostasis and that mice that are constitutively LC deficient are predisposed to develop augmented T-cell responses on that basis. However, to date, there is no evidence for perturbed immune homeostasis in Langerin-DTA mice, and a mechanism that might promote such an effect has not been identified.

\section{CONCLUSION AND FUTURE PROSPECTS}

The in vivo mouse studies that we have briefly summarized have clearly provided new insights into skin DC biology. It is interesting to note that, to date, the results of these studies have introduced complexities into the picture more often than they have brought clarity. As the studies reported thus far have emphasized the steady-state condition and defined acute inflammatory stimuli, it can be anticipated that additional complexities will become apparent as chronic infections and/or chronic inflam- matory conditions are studied in mice and in man. It seems likely that approaches that target additional skin accessory cell populations in mice as they are defined will also be fruitful.

Experience with CD11c-DTR mice ${ }^{32}$ suggests that future studies may be challenging, however, and it is possible that the successes with Langerin-DTA and Langerin-DTR mice may not be predictive. As all murine DCs express the $\beta 2$ integrin CD11c, it was anticipated that DC in CD11c-DTR mice would selectively be depleted by administering DT. In initial experiments, it was determined that although DT killed most DCs, some $\mathrm{CD} 11 \mathrm{c}+$ sub-populations (including LC) were resistant. In addition, DCs that were depleted reappeared within a few days and repeated DT treatment was lethal. In these mice, DC function could only be assessed in very acute experiments. Subsequent experiments involving bone marrow chimeras revealed that the lethality resulted from effects of DT on nonhematopoetic cells in CD11c-DTR mice. Leukocytes expressing CD11c could be successfully depleted from chimeric mice (CD11c-DTR bone marrow into wild-type recipients) with DT, but depletion of some populations is incomplete and CD11c is also expressed by some non-DC. All of these confounding findings have limited the utility of CD11c-DTR mice to some extent. Thus, although the approach is powerful, the degree to which future experiments are informative will hinge on identification of proteins and genes that are selectively expressed at relatively high levels by the subpopulations that are to be targeted. This kind of information will come from observational studies that continue to delve into skin accessory cell heterogeneity. The field is moving ahead at a rapid pace, but many mysteries remain to be solved.

\section{ACKNOWLEDGMENTS}

We thank Miriam Merad for helpful discussions and for allowing us to review and cite materials that were in press when this paper was prepared. This study was supported by the Intramural Program of the $\mathrm{NIH}$, Center for Cancer Research, NCl.

\section{DISCLOSURE}

The authors declared no conflict of interest.

(c) 2008 Society for Mucosal Immunology

\section{REFERENCES}

1. Kissenpfenning, A. \& Malissen, B. Langerhans cells - revisiting the paradigm using genetically engineered mice. Trends Immunol. 27, 132139 (2006).

2. Shortman, K. \& Naik, S.H. Steady-state and inflammatory dendritic-cell development. Nat. Rev. Immunol. 7, 19-30 (2007).

3. Merad, M., Ginhoux, F. \& Collin, M. Origin, homeostasis and function of human and mouse langerin+ dendritic cells. Nat. Rev. Immunol. (in press).

4. Katz, S.I., Tamaki, K. \& Sachs, D.H. Epidermal Langerhans cells are derived from cells originating in bone marrow. Nature 282, 324-326 (1979).

5. Frelinger, J.G., Hood, L., Hill, S. \& Frelinger, J.A. Mouse epidermal la molecules have a bone marrow origin. Nature 282, 321-323 (1979).

6. Schuler, G. \& Steinman, R.M. Murine epidermal Langerhans cells mature into potent immunostimulatory dendritic cells in vitro. J. Exp. Med. 161, 526-546 (1985).

7. Banchereau, J. \& Steinman, R.M. Dendritic cells and the control of immunity. Nature 392, 245-52 (1998).

8. Steinman, R.M. \& Nussenzweig, M.C. Avoiding horror autotoxicus: the importance of dendritic cells in peripheral T cell tolerance. Proc. Natl. Acad. Sci. USA 99, 351-358 (2002). 
9. Kissenpfenning, A. et al. Disruption of the langerin/CD207 gene abolishes Birbeck granules without a marked loss of Langerhans cell function. Mol. Cell. Biol. 25, 88-99 (2005).

10. Kaplan, D.H. et al. Epidermal Langerhans cell-deficient mice develop enhanced contact hypersensitivity. Immunity 23, 611-620 (2005).

11. Kissenpfenning, A. et al. Dynamics and function of Langerhans cells in vivo: dermal dendritic cells colonize lymph node areas distinct from slower migrating Langerhans cells. Immunity 22, 643-654 (2005).

12. Bennett, C.L. et al. Inducible ablation of mouse Langerhans cells diminishes but fails to abrogate contact hypersensitivity. J. Cell. Biol. 169, 569-576 (2005).

13. Ginhoux, F. et al. Blood-derived dermal langerin dendritic cells survey the skin in the steady state. J. Exp. Med. 204, 3133-3146 (2007).

14. Bursch, L.S. et al. Identification of a novel population of Langerin+ dendritic cells. J. Exp. Med. 204, 3147-3156 (2007)

15. Poulin, L.F. et al. The dermis contains langerin+ dendritic cells that develop and function independently of epidermal Langerhans cells. J. Exp. Med. 204, 3119-3131 (2007).

16. Borkowski, T.A., Nelson, A.J., Farr, A.G. \& Udey, M.C. Expression of gp40, the murine homologue of human epithelial cell adhesion molecule (Ep-CAM), by murine dendritic cells. Eur. J. Immunol. 26, 110-114 (1996).

17. Merad, M. et al. Langerhans cells renew in the skin throughout life under steady-state conditions. Nat. Immunol. 3, 1135-1141 (2002).

18. Merad, M. et al. Depletion of host Langerhans cells before transplantation of donor alloreactive T cells prevents skin graft-versus-host disease. Nat. Med. 10, 510-517 (2004).

19. Borkowski, T.A., Letterio, J.J., Farr, A.G. \& Udey, M.C. A role for endogenous transforming growth factor beta 1 in Langerhans cell biology: the skin of transforming growth factor beta 1 null mice is devoid of epidermal Langerhans cells. J. Exp. Med. 184, 2417-2422 (1996).
20. Borkowski, T.A. et al. A role for TGFbeta1 in Langerhans cell biology. Further characterization of the epidermal Langerhans cell defect in TGFbeta1 null mice. J. Clin. Invest. 100, 575-581 (1997).

21. Kaplan, D.Het al. Autocrine/paracrine TGF $\beta 1$ is required for the development of epidermal Langerhans cells. J. Exp. Med. 204, 25452552 (2007).

22. Bogunovic, M. et al. Identification of a radio-resistant and cycling dermal dendritic cell population in mice and men. J. Exp. Med. 203, 2627-2638 (2006).

23. Ginhoux, F. et al. Langerhans cells arise from monocytes in vivo. Nat. Immunol. 7, 265-273 (2006).

24. Larregina, A.T. et al. Dermal-resident CD14+ cells differeniate into Langerhans cells. Nat. Immunol. 2, 1151-1158 (2001).

25. Cheong, C. et al. Production of monoclonal antibodies that recognize the extracellular domain of mouse langerin/CD207. J. Immunol. Methods 324, 48-62 (2007).

26. Strid, J. et al. Acute upregulation of an NKG2D ligand promotes rapid reorganization of a local immune compartment with pleiotropic effects on carcinogenesis. Nat. Immunol. 9, 146-154 (2008).

27. Obhrai, J.S. et al. Langerhans cells are not required for efficient skin graft rejection. J. Invest. Dermatol. 128, 1950-1955 (2008).

28. Stoecklinger, A. et al. Epidermal Langerhans cells are dispensable for humoral and cell-mediated immunity elicited by gene gun immunization. J. Immunol. 179, 886-893 (2007).

29. Bennett, C.L. et al. Langerhans cells are required for efficient presentation of topically applied hapten to T cells. J. Immunol. 179, 6830-6835 (2007).

30. Stoitzner, P. et al. Tumor immunotherapy by epicutaneous immunization requires Langerhans cells. J. Immunol. 180, 1991-1998 (2008).

31. Wang, L. et al. Langerin expressing cells promote skin immune responses under defined conditions. J. Immunol. 180, 4722-4727 (2008).

32. Bennett, C.L. \& Clausen, B.E. DC ablation in mice: promises, pitfalls, and challenges. Trends Immunol. 28, 525-531 (2007). 